\title{
Flexible bronchoscopy may decrease respiratory muscle strength: premedicational midazolam in focus
}

Baykal Tulek*, Fikret Kanat, Sule Tol and Mecit Suerdem

\begin{abstract}
Background: Flexible bronchoscopy (FB) is a procedure accepted to be safe in general, with low complication rates reported. On the other hand, it is known that patients with pre-existing respiratory failure have developed hypoventilation following FB. In this study the effects of FB on respiratory muscle strength were investigated by measuring maximum respiratory pressures.

Methods: One hundred and forty patients, aged between 25 and 90 years, who had undergone diagnostic bronchoscopy between February 2012 and May 2012, were recruited to the study. Pre- and post-procedure maximal inspiratory pressure (MIP) and maximal expiratory pressure (MEP) were measured. A correlation between the MIP and MEP changes and patient characteristics and FB variables were investigated.

Results: Significant decreases in both MIP and MEP values were observed following FB ( $p<0.001$ for both). Decreases were attributed to the midazolam used for sedation. Significant decreases in respiratory muscle strengths were observed especially in the high-dose midazolam group, compared to both low-dose and non-midazolam groups.

Conclusions: It was determined that respiratory muscle weakness may arise post-procedure in patients who have undergone $\mathrm{FB}$, and this is constitutively related to midazolam premedication. Respiratory muscle weakness might play a role in potential hypoventilation in critical patients who undergo FB.
\end{abstract}

Keywords: Bronchoscopy, Midazolam, Respiratory muscle strength

\section{Background}

Flexible bronchoscopy $(\mathrm{FB})$ is a procedure that is widely used in the diagnosis and management of airway and lung diseases, and is accepted to be safe in general [1]. Data regarding bronchoscopy complications and low complication rates are mostly obtained from retrospective studies. However, the increase in therapeutic bronchoscopy applications, which are becoming more and more complex, and the bronchoscopy applications in risky patient groups cause an increase in complication rates [2].

Hypoventilation has been shown to develop during and after the procedure in patients who undergo bronchoscopy $[3,4]$. This has mostly been linked to sedative

\footnotetext{
* Correspondence: baykaltulek@yahoo.com

Selcuk University, Selcuklu Faculty of Medicine, Department of Chest Diseases, Selçuklu 42075, Konya, Turkey
}

agents in relevant studies. However, to our knowledge, the effects of bronchoscopy itself and/or sedative agents on respiratory muscle strength have yet to be studied. The aim of this study was to evaluate the effects of bronchoscopy on respiratory muscle strength, which as an important role in respiratory function and induction of cough reflex $[5,6]$.

\section{Methods \\ Patients}

One hundred and forty patients, aged between 25 and 90 years, who had undergone diagnostic bronchoscopy between February 2012 and May 2012 were recruited to the study. Patients with hemodynamic instability (heart rate below $50 \mathrm{bpm}$ or above $120 \mathrm{bpm}$, systolic arterial blood pressure below $90 \mathrm{mmHg}$ or above $180 \mathrm{mmHg}$ ), basal oxygen saturation $<90 \%, \mathrm{PaCO}_{2}>45 \mathrm{mmHg}$, liver 
or kidney dysfunction, those who were diagnosed with a neuromuscular disease, had cancer with cachexia, medium $\left(\mathrm{FEV}_{1}\right.$ between 50 and $\left.80 \%\right)$, severe $\left(\mathrm{FEV}_{1}\right.$ between 30 and $50 \%)$ or very severe $\left(\mathrm{FEV}_{1}<30 \%\right) \mathrm{COPD}$, and those who could not cooperate with respiratory muscle strength test or with benzodiazepine allergy have not been included into the study. Study protocol was approved by Selcuk University Selcuklu Medical Faculty Ethics Committee. Written informed consent was obtained from all participants.

\section{Bronchoscopy}

All bronchoscopy procedures were performed by the same experienced investigator in accordance with the international guidelines [7]. In the absence of any contraindications, IM atropine $(0.01 \mathrm{mg} / \mathrm{kg})$ was given to the patients half an hour prior to the procedure. $2 \%$ lidocaine was applied topically to the inside of the mouth, pharynx, upper respiratory airways and endobronchially. As a routine application at our clinic, IV midazolam was offered to all patients by the bronchoscopist prior to the procedure and $1 \mathrm{mg}$ IV midazolam was given to those who accepted the offer 3 minutes prior to the procedure. While no additional doses were given to patients who had adequate sedation during the procedure, additional doses of $1 \mathrm{mg}$ were given in 2-minute intervals to patients considered to be in need by the bronchoscopist. At the end of the study, patients were retrospectively separated into three groups: no midazolam (Group A), low-dose midazolam (Group B), and high-dose midazolam (Group C).

$2-4 \mathrm{ml} / \mathrm{min}$ oxygen supplementation was performed in all patients, and oxygen flow was increased in case of hypoxemia. Flumazenil was kept available for suspected cases of respiratory depression. An IV vascular access was prepared, and electrocardiogram (ECG), oxygen saturation and non-invasive blood pressure monitoring were performed throughout the procedure. Total duration of bronchoscopy, midazolam dose and adverse events during the endoscopic procedure were recorded. Cardiopulmonary complications observed during the flexible bronchoscopy were defined as the following: hypotension (systolic blood pressure (BP) $<90 \mathrm{mmHg}$ or mean arterial blood pressure $<60 \mathrm{mmHg}$ ), hypertension (systolic BP $>180 \mathrm{mmHg}$ or diastolic BP $>90 \mathrm{mmHg}$ ), tachycardia (HR $>100 / \mathrm{min}$ and/or a variation of $>20 \%$ from baseline value), bradycardia (HR $<50 / \mathrm{min}$ ), and oxygen desaturation $\left(\mathrm{SaO}_{2}\right.$ decrease $<90 \%$ for $>30$ s). Monitoring was continued for 2 hours following the procedure. 0-100 mm visual analogue scale (VAS) was used two hours after the flexible bronchoscopy to investigate the comfort of the procedure. A higher score meant better satisfaction or less discomfort (0: worst imaginable health state, 100: best imaginable health state).

\section{Maximum respiratory pressure measurements}

A hand-held respiratory pressure meter (Micro RPM; Micro Medical, Chatham, UK) was used to measure respiratory muscle strength [8]. Maximal inspiratory pressure (MIP) was acquired from residual volume (RV) and the maximal expiratory pressure (MEP) was acquired from total lung capacity (TLC). Measurements were taken prior to bronchoscopy before premedication and after 30 minutes following the procedure by the same investigator without any knowledge of the clinical conditions of the patients. The operator explained the procedure before beginning and showed the correct manoeuvre. MIP was measured first, followed by MEP. Patients performed 5 maneuvers in intervals of 1 minute for each measurement and the highest values ( $<20 \%$ variance) were recorded [9].

\section{Statistical analyses}

Descriptive statistics are shown as mean $( \pm S D)$ or count (percentage). $T$-test was used to compare respiratory muscle strengths before and after bronchoscopy for all patients. T-test or One-Way ANOVA were used to compare each variable for the mean MIP and MEP changes. One-Way ANOVA and Chi-square tests were utilized in the comparison of continuous and categorical variables between various midazolam dose groups, respectively. The post-hoc comparisons of significant difference between the groups were performed using the Tukey test. The statistical significance level was set to 0.05 .

\section{Results}

Bronchoscopies were successfully performed in all of the 140 patients included in the study. As to the midazolam dosages: 34 patients did not want midazolam administration (Group A), 66 were administered $1 \mathrm{mg}$ of midazolam prior to the procedure, and 40 received high doses (mean, $0.05 \pm 0.03 \mathrm{mg} / \mathrm{kg}$ ) of midazolam (Group C). Patient characteristics and complications developed during the bronchoscopy are presented in Table 1 . The study population mostly comprised middle- and oldaged males. One third of the patients had mild COPD and the most common complication observed was tachycardia. On the contrary, hypertension, bradycardia, hypotension and desaturation were rarely encountered complications.

When the overall pre- and post-procedure MIP and MEP values were compared $\left(73.9 \pm 24.4 \quad \mathrm{cmH}_{2} \mathrm{O}\right.$ vs $68.9 \pm 25.2 \quad \mathrm{cmH}_{2} \mathrm{O} ; 99.9 \pm 29.8 \mathrm{cmH}_{2} \mathrm{O}$ vs. $89.9 \pm 28.1$ $\mathrm{cmH}_{2} \mathrm{O}$ respectively), significant decreases in both values were observed ( $p<0.001$ for both comparisons; Figure 1).

Patients were dichotomized according to median age, median duration of bronchoscopy, smoking status, and COPD history in order to determine the variables that may cause a decrease in respiratory muscle strength. In 
Table 1 Demographics and clinical and bronchoscopic characteristics of patients

\begin{tabular}{ll}
\hline Variables & All patients $(\mathbf{n}=\mathbf{1 4 0})$ \\
\hline Age, $y$ & $58.1 \pm 13.5$ \\
\hline Male gender, $n(\%)$ & $102(72.9)$ \\
\hline Patients with COPD, $n(\%)$ & $44(31.4)$ \\
\hline History of smoking, $n(\%)$ & $73(52.1)$ \\
\hline Smoking pack years & $38.4 \pm 22.4$ \\
\hline Duration of bronchoscopy (min) & $15.9 \pm 6.1$ \\
\hline Patient comfort (VAS mm) & $78.8 \pm 17.1$ \\
\hline Midazolam groups, $n$ & \\
\hline Non & 34 \\
\hline Low-dose & 66 \\
\hline High-dose & 40 \\
\hline Complications, $n$ (\%) \\
\hline Tachycardia & $62(44.3)$ \\
\hline Bradycardia & $2(1.4)$ \\
\hline Hypotension & $1(0.7)$ \\
\hline Hypertension & $13(9.3)$ \\
\hline Desaturation & $4(2.9)$ \\
\hline
\end{tabular}

addition, the pre- and post-procedure MIP and MEP values were investigated between the different midazolam dose groups as well. Among these variables, a significant difference was determined only in different midazolam dose groups in terms of $\Delta$ MIP and $\triangle$ MEP (Table 2).

The post-hoc analysis in the midazolam groups revealed no significant difference between the Groups A and $\mathrm{B}$ in terms of $\Delta$ MIP $(\mathrm{p}>0.05)$ while $\Delta$ MIP was higher in Group $\mathrm{C}$ when compared to Group A $(\mathrm{p}<0.001)$ and Group B $(\mathrm{p}<0.001)$. There was no significant difference between Group A and Group B in terms of $\triangle$ MEP $(p>0.05)$. $\triangle$ MEP was higher in Group $C$ when compared to Groups A $(\mathrm{p}=0.04)$ and B $(\mathrm{p}=0.05)$.

The comparison of pre- and post-procedure MIP and MEP values among different midazolam dose groups using $T$-test revealed no significant difference in terms of MIP measurements in the Groups A and B; however, there was a significant decrease in post-procedure MEP values in both groups. Significant decreases in both the post-procedure MIP and MEP values were determined in Group C (Table 3).

No significant differences were determined in terms of age, sex, smoking status, ratio of patients with COPD, duration of bronchoscopy, and basal MIP/MEP measurements in different midazolam groups. However, there was a significant difference between the groups in terms of patient comfort assessed using VAS ( $<<0.001)$, and while there was no significant difference between the Groups A and B in the post-hoc test, the VAS scores of Group $C$ was determined to be higher than the patients in Group A and Group B ( $<<0.001$ for both comparisons; Table 4).

\section{Discussion}

In this study, where -to our knowledge- the effects of FB and premedication on respiratory muscle strengths were investigated for the first time, it was revealed that midazolam, administered for sedation purposes in bronchoscopy, might affect negatively the respiratory muscle strengths, as shown with MIP and MEP measurements.

Flexible bronchoscopy is generally regarded as a safe procedure [1]. Hypoventilation is one of the most common complications and many causes of hypoventilation related to FB have been defined. These include ventilation-perfusion mismatch related to the procedure itself, upper airway obstruction, sedation-related central respiratory depression, and increased resistance due to introduction of bronchoscope into the trachea $[3,10]$. Our findings show that sedation-related respiratory muscle weakness can be included among these mechanisms.

Pulse oxymetry is utilized in many centers for monitoring hypoventilation during bronchoscopy. On the other hand, patients are routinely given oxygen during FB in many centers. Bronchoscopy guidelines recommend oxygen supplementation to maintain oxygen saturation at a minimum of $90 \%$, because it will reduce the risk of arrhythmia during and after bronchoscopy [7]. However, even though severe $\mathrm{CO}_{2}$ retention may occur during the procedure, oxyhemoglobin desaturation may not be observed. Chhajed et al. [3] have performed cutaneous carbon dioxide tension $\left(\mathrm{PcCO}_{2}\right)$ measurements in addition to the oxymetry in patients that underwent $\mathrm{FB}$ in which sedation was achieved with intermittent intravenous midazolam and $5 \mathrm{mg}$ of hydrocodone, and determined an increase in $\mathrm{PcCO}_{2}$ in all but one patient. The highest $\mathrm{PcCO}_{2}$ value was significantly associated to the

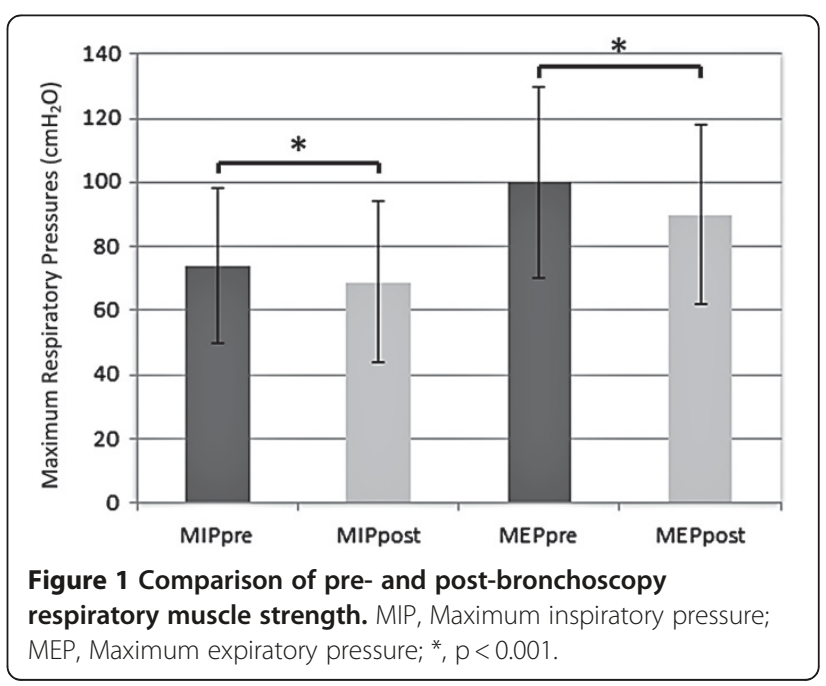


Table 2 Comparison of the differences in pre- and post-procedure maximum respiratory pressures ( $\Delta$ : pre-post) between different patient groups

\begin{tabular}{|c|c|c|c|c|c|}
\hline & \multirow[t]{2}{*}{$N$} & \multicolumn{2}{|c|}{$\Delta$ MIP (pre-post) } & \multicolumn{2}{|c|}{$\Delta$ MEP (pre-post) } \\
\hline & & Mean \pm SD $\left(\mathrm{cmH}_{2} \mathrm{O}\right)$ & $p$ & Mean \pm SD $\left(\mathrm{cmH}_{2} \mathrm{O}\right)$ & $\mathrm{p}$-value \\
\hline \multicolumn{6}{|l|}{ Age (years) } \\
\hline$<60$ & 69 & $2.7 \pm 16.4$ & NS & $10.0 \pm 22.61$ & NS \\
\hline$\geq 60$ & 71 & $7.2 \pm 14.8$ & & $10.0 \pm 15.7$ & \\
\hline \multicolumn{6}{|l|}{ Smoking } \\
\hline Non-smoker & 67 & $6.2 \pm 16.5$ & NS & $9.8 \pm 14.8$ & NS \\
\hline Smoker & 73 & $3.9 \pm 14.9$ & & $10.2 \pm 22.7$ & \\
\hline \multicolumn{6}{|l|}{ COPD } \\
\hline No & 96 & $5.4 \pm 15.7$ & NS & $11.2 \pm 21.4$ & NS \\
\hline Yes & 44 & $4.2 \pm 15.9$ & & $7.4 \pm 13.5$ & \\
\hline \multicolumn{6}{|c|}{ Duration of bronchoscopy (min) } \\
\hline$<15.5$ & 69 & $4.5 \pm 16.6$ & NS & $10.2 \pm 20.0$ & NS \\
\hline$\geq 15.5$ & 71 & $5.5 \pm 15.0$ & & $9.9 \pm 18.8$ & \\
\hline \multicolumn{6}{|c|}{ Midazolam group } \\
\hline $\mathrm{A}$ & 34 & $0.4 \pm 16.0$ & $<0.001$ & $6.2 \pm 16.0$ & $<0.05$ \\
\hline $\mathrm{B}$ & 66 & $1.8 \pm 13.4$ & & $7.8 \pm 17.6$ & \\
\hline$C$ & 40 & $14.2 \pm 15.6^{*}$ & & $16.8 \pm 22.9^{* *}$ & \\
\hline
\end{tabular}

*Via post-hoc analysis, pair-wised comparison of Group C with both Group A and B ( $p<0.001$ for both). ** Via post-hoc analysis, pair-wised comparison of Group C with Group A $(p=0.04)$ and Group B $(p=0.05)$.

baseline $\mathrm{PcCO}_{2}(\mathrm{p}<0.0001)$ and lowest $\mathrm{SpO}_{2}(\mathrm{p}=0.016)$. Dreher et al. [4] have utilized $\mathrm{PcCO}_{2}$ measurements in order to evaluate alveolar hypoventilation in patients with pre-existing respiratory failure, and determine a significant increase in $\mathrm{PcCO}_{2}$ during the procedure. In their study, while no significant difference was determined in terms of $\mathrm{PCCO}_{2}$ during $\mathrm{FB}$ between the groups where the patients were sedated with either midazolam alone or midazolam plus alfentanil, $\mathrm{PCCO}_{2}$ was higher compared to baseline in the midazolam alone group 120 minutes after the procedure than the midazolam plus alfentanil group. The cause for prolonged hypoventilation was considered to be midazolam due to the fact that the midazolam alone group received twice the amount of midazolam than the midazolam plus alfentanil group ( $4 \mathrm{mg}$ vs. $2 \mathrm{mg}$ ). We believe that the decrease in both the inspiratory and expiratory respiratory muscle strengths, which was determined in the high-dose $(0.05 \pm 0.03 \mathrm{mg} / \mathrm{kg})$ midazolam group in our study, might play an important role in hypoventilation that has been determined in the two studies mentioned above. However, because we did not measure $\mathrm{PcCO}_{2}$ in our study, we do not know whether there was an increase in the $\mathrm{CO}_{2}$ values or not, and thus, we do not know whether the determined muscle weakness has any clinical importance or not.

The effects of midazolam on respiratory muscle strength have been previously shown in experimental and clinical studies. Fujii et al. [11] have investigated the effects of midazolam and propofol on diaphragm contractility in dogs. In that study, they have induced diaphragmatic fatigue with intermittent supramaximal bilateral electrophrenic stimulation at low or high frequency (20 and $100 \mathrm{~Hz}$, respectively), and after the induction of fatigue, in order to assess the diaphragm contractility, transdiaphragmatic pressure (Pdi) and integrated electrical activity of the crural $\left(\mathrm{E}_{\mathrm{di}-\mathrm{cru}}\right)$ and costal $\left(E_{\text {di-cost }}\right)$ parts of the diaphragm were measured. Their findings showed that midazolam caused a decrease in Pdi at both frequencies when compared to fatigued values $(\mathrm{p}<0.05)$, and that $\mathrm{E}_{\mathrm{di}-\text { cru }}$ and $\mathrm{E}_{\mathrm{di}-\text { cost }}$ at $100 \mathrm{~Hz}$ stimulation during midazolam administration were below the baseline values $(p<0.05)$. They have also reported a lower Pdi value in the midazolam group than in the propofol group $(\mathrm{p}<0.05)$.

Table 3 Comparison of pre- and post-flexible bronchoscopy MIP and MEP values in different midazolam dose groups

\begin{tabular}{|c|c|c|c|c|c|c|}
\hline & \multicolumn{2}{|c|}{ MIP $\left(\mathrm{cm} \mathrm{H}_{2} \mathrm{O}\right)$} & \multirow[t]{2}{*}{$p$} & \multicolumn{2}{|c|}{ MEP $\left(\mathrm{cm} \mathrm{H}_{2} \mathrm{O}\right)$} & \multirow[t]{2}{*}{$P$} \\
\hline & Pre & Post & & Pre & Post & \\
\hline Group A & $80.5 \pm 25.2$ & $80.0 \pm 24.7$ & NS & $109.4 \pm 27.6$ & $103.2 \pm 21$ & $=0.03$ \\
\hline Group B & $69.3 \pm 23.1$ & $67.6 \pm 22.8$ & NS & $93.6 \pm 30.3$ & $85.8 \pm 29.0$ & $=0.001$ \\
\hline Group C & $75.9 \pm 24.9$ & $61.7 \pm 26.6$ & $<0.001$ & $102.1 \pm 28.9$ & $85.3 \pm 28.9$ & $<0.001$ \\
\hline
\end{tabular}


Table 4 Comparison regarding patient demographics, bronchoscopic characteristics and pre-procedure maximum respiratory pressures among different midazolam dose groups

\begin{tabular}{|c|c|c|c|c|}
\hline & Group A (n: 34) & Group B (n: 66) & Group C (n: 40) & $\mathrm{p}$ \\
\hline Male gender, n (\%) & $24(70.6)$ & $53(80.3)$ & $25(62.5)$ & NS \\
\hline Age & $58.9 \pm 11.8$ & $55.9 \pm 14.5$ & $60.9 \pm 12.8$ & NS \\
\hline Patients with COPD, n (\%) & $14(41.2)$ & $21(31.8)$ & $9(22.5)$ & NS \\
\hline Smoking pack years & $27.6 \pm 11.6$ & $38.9 \pm 18.9$ & $36.9 \pm 21.9$ & NS \\
\hline Duration of bronchoscopy (min) & $15.5 \pm 5.9$ & $15.6 \pm 5.4$ & $16.9 \pm 7.3$ & NS \\
\hline \multicolumn{5}{|l|}{ Complications } \\
\hline Tachycardia & 19 & 31 & 12 & NS \\
\hline Bradycardia & 1 & 1 & 0 & NS \\
\hline Hypotension & 0 & 1 & 0 & NS \\
\hline Hypertension & 5 & 5 & 3 & NS \\
\hline Desaturation & 2 & 1 & 1 & NS \\
\hline Patient comfort & $76.2 \pm 17.4$ & $74.4 \pm 17.4$ & $88.1 \pm 12.5^{*}$ & $<0.001$ \\
\hline MIP $1\left(\mathrm{cmH}_{2} \mathrm{O}\right)$ & $80.5 \pm 25.2$ & $69.3 \pm 23.1$ & $75.9 \pm 24.9$ & NS \\
\hline MEP 1 & $109.4 \pm 27.6$ & $93.6 \pm 30.1$ & $102.1 \pm 28.9$ & NS \\
\hline
\end{tabular}

* When compared to Groups A and B using the post-hoc test: $p<0.001$.

In another study, the effects of sedative $(0.1 \mathrm{mg} / \mathrm{kg} / \mathrm{h})$ and anesthetic $(0.5 \mathrm{mg} / \mathrm{kg} / \mathrm{h})$ dosages of midazolam on the decrease in diaphragm contractility, fatigue (detail fatigue rating [DFR]), have been evaluated in dogs [12]. They showed that an infusion of midazolam has caused a decrease from baseline values $(\mathrm{p}<0.05)$ in Pdi at 20 and $100 \mathrm{~Hz}$ stimulations, and that $\% \mathrm{E}_{\mathrm{di}-\mathrm{cru}}$ and $\% \mathrm{E}_{\mathrm{di}-\text { cost }}$ values at $100 \mathrm{~Hz}$ were below baseline $(\mathrm{p}<0.05)$ in both sedative and anesthetic groups. They have also demonstrated that the Pdi and $\% \mathrm{E}_{\mathrm{di}}$ decrease was greater in the anesthetic dose group than in the sedative dose group $(\mathrm{p}<0.05)$. Their findings show that contractility of fatigued diaphragm dose-dependently decreases with midazolam.

Molliex et al. [13] have studied the effects of midazolam, with a dosage of $0.1 \mathrm{mg} / \mathrm{kg}$, on total pulmonary resistance and diaphragmatic, intercostal and abdominal muscle patterns in 9 healthy volunteers. Changes in gastric pressure $(\Delta \mathrm{Pga})$ and pleural pressure $(\Delta \mathrm{Ppl})$ were measured in all participants, and the reduction in diaphragm contractility was evaluated with $\Delta \mathrm{Pga} / \Delta \mathrm{Ppl}$. Midazolam was determined to increase total pulmonary resistance in sedative doses, and associatively, an increase in intercostal muscle activity was also determined; however, the diaphragmatic contribution to respiratory process was found to be decreased. This was explained as a shift from an abdominal breathing to predominantly rib cage breathing rather than a decrease in diaphragm contractility. Even though upper airway measurements were not taken in the current study, the increase in pulmonary resistance is thought to be associated with upper airway occlusion. Diazepam causing a decrease in the activity of the genioglossus muscle, which has an important role in maintaining the patency of the upper airway, supports this hypothesis [14].

It is known that bronchoscopy deleteriously affects pulmonary mechanics and lung volumes $[10,15]$. This may arise a question regarding whether the significant reductions in maximal pressures are related to lung volumes rather than muscle weakness or respiratory muscle strength. MIP is measured at or close to RV and MEP at or close to TLC. Sometimes these measurements were performed at functional residual capacity. Although the latter may be more accurate for some studies, in that case the lung volumes should be specifically stated [16]. In patients with abnormally high lung volumes, a low MIP may partly reflect the shortened inspiratory muscle fiber length associated with increased lung volume at RV rather than reduced inspiratory muscle strength; however, in our study all moderate to very severe COPD patients were not taken into the study and also the groups were not different regarding the number of mild COPD patients. Therefore it may be considered that the reductions in maximal pressures are probably not related to lung volumes.

Although there is limited falls in MEP values in patients who were not sedated during bronchoscopy, when $\triangle$ MEP values were compared between groups, the post-hoc analysis revealed that the difference was statistically significant only with group $\mathrm{A}$ and $\mathrm{C}$ and the decline in MEP values was higher in the high-dose midazolam group. When pre and post MEP values within each group were compared, post MEP values in group B and $\mathrm{C}$ were significantly lower than group $\mathrm{A}$. It is notable that although post MIP values were significantly lower only in Group C, there were remarkable falls in post 
MEP values in all groups. The fall in post MEP values even in group A, i.e. in groups who were not sedated with midazolam might be due to exhaustion of the patient after repeated maneuvers for correct MIP measurements.

Even though the overall bronchoscopy comfort scores were quite high in our study, the fact of significantly higher VAS scores of the patients in the high-dose rather than in the low-dose midazolam group, and of the similarity of the patient comfort levels in the low-dose and no midazolam groups can be explained by the bronchoscopist's tendency to administer low doses. It is known that objective techniques, such as electroencephalogrambased bispectral index [17] or Ramsay sedation score [18], allow a more effective titration of the sedatives. In addition, it is also shown that both inter-individual and intra-individual variations are seen in the online sedation monitoring in healthy volunteers who were sedated with midazolam [19]. This condition may explain why some patients needed high doses and some low doses of midazolam in our study although the bronchoscopist decided the appropriate sedation level in patients subjectively. In humans, midazolam is mostly metabolized by CYP3A 45 isozymes to one major metabolite, 1-hydroxymidazolam and to some extent to 4-hydroxymidazolam and 1,4dihydroxymidazolam [20,21]. It is well known that wide inter-individual variations in hepatic and intestinal CYP3A activity are seen in the human population [22].

\section{Conclusion}

In conclusion, midazolam for premedication purposes has been determined to increase comfort in patients undergoing flexible bronchoscopy, however, causing significant decrease in respiratory muscle strength. This might increase the probability of possible complications following bronchoscopy in critical patients who have advanced COPD with limited respiratory reserves or respiratory insufficiency. Certainly there is a special need for further studies with risk patient groups and the use of high-dose midazolam.

\section{Competing interests}

The authors of the paper "Flexible bronchoscopy may decrease respiratory muscle strength: premedicational midazolam in focus" declare to have no competing interests that might be related to the contents of the manuscript.

\section{Authors' contribution}

BT helped design the study, conduct of the study, collection of data, analysis of data and prepare the manuscript. FK helped conduct of the study, collection of data, analysis of data and prepare the manuscript. ST helped conduct of the study, collection of data. MS helped conduct of the study, collection of data, and prepare the manuscript. All authors read and approved the final manuscript.

Received: 12 July 2012 Accepted: 7 September 2012 Published: 25 September 2012

\section{References}

1. Facciolongo N, Patelli M, Gasparini S, Lazzari Agli L, Salio M, Simonassi C, Del Prato B, Zanoni P: Incidence of complications in bronchoscopy.
Multicentre prospective study of 20,986 bronchoscopies. Monaldi Arch Chest Dis 2009, 71(1):8-14.

2. Jin F, Mu D, Chu D, Fu E, Xie Y, Liu T: Severe complications of bronchoscopy. Respiration 2008, 76(4):429-433.

3. Chhajed PN, Rajasekaran R, Kaegi B, Chhajed TP, Pflimlin E, Leuppi J, Tamm M: Measurement of combined oximetry and cutaneous capnography during flexible bronchoscopy. Eur Respir J 2006, 28(2):386-390.

4. Dreher M, Ekkernkamp E, Storre JH, Kabitz HJ, Windisch W: Sedation during flexible bronchoscopy in patients with pre-existing respiratory failure: Midazolam versus Midazolam plus Alfentanil. Respiration 2010, 79(4):307-314

5. Begin P, Grassino A: Inspiratory muscle dysfunction and chronic hypercapnia in chronic obstructive pulmonary disease. Am Rev Respir Dis 1991, 143(5 Pt 1):905-912.

6. Park JH, Kang SW, Lee SC, Choi WA, Kim DH: How respiratory muscle strength correlates with cough capacity in patients with respiratory muscle weakness. Yonsei Med J 2010, 51(3):392-397.

7. British Thoracic Society guidelines on diagnostic flexible bronchoscopy. Thorax 2001, 56(1):1-21.

8. Dimitriadis Z, Kapreli E, Konstantinidou I, Oldham J, Strimpakos N: Test/retest reliability of maximum mouth pressure measurements with the MicroRPM in healthy volunteers. Respir Care 2011, 56(6):776-782.

9. Evans JA, Whitelaw WA: The assessment of maximal respiratory mouth pressures in adults. Respir Care 2009, 54(10):1348-1359.

10. Matsushima $Y$, Jones RL, King EG, Moysa G, Alton JD: Alterations in pulmonary mechanics and gas exchange during routine fiberoptic bronchoscopy. Chest 1984, 86(2):184-188.

11. Fujii $Y$, Toyooka $H$ : Midazolam versus propofol for reducing contractility of fatigued canine diaphragm. Br J Anaesth 2001, 86(6):879-881.

12. Fujii $Y$, Uemura A, Toyooka H: Midazolam-induced muscle dysfunction and its recovery in fatigued diaphragm in dogs. Anesth Analg 2003, 97(3):755-758

13. Molliex S, Dureuil B, Montravers $P$, Desmonts JM: Effects of midazolam on respiratory muscles in humans. Anesth Analg 1993, 77(3):592-597.

14. Leiter JC, Knuth SL, Krol RC, Bartlett D Jr: The effect of diazepam on genioglossal muscle activity in normal human subjects. Am Rev Respir Dis 1985, 132(2):216-219.

15. Peacock AJ, Benson-Mitchell R, Godfrey R: Effect of fibreoptic bronchoscopy on pulmonary function. Thorax 1990, 45(1):38-41.

16. ATS/ERS Statement on respiratory muscle testing. Am J Respir Crit Care Med 2002, 166(4):518-624.

17. Bower AL, Ripepi A, Dilger J, Boparai N, Brody FJ, Ponsky JL: Bispectral index monitoring of sedation during endoscopy. Gastrointest EndosC 2000, 52(2):192-196.

18. Ramsay MA, Savege TM, Simpson BR, Goodwin R: Controlled sedation with alphaxalone-alphadolone. Br Med J 1974, 2(5920):656-659.

19. Haenggi M, Ypparila-Wolters H, Hauser K, Caviezel C, Takala J, Korhonen I, Jakob SM: Intra- and inter-individual variation of BIS-index and Entropy during controlled sedation with midazolam/remifentanil and dexmedetomidine/remifentanil in healthy volunteers: an interventional study. Crit Care 2009, 13(1):R20

20. Kronbach T, Mathys D, Umeno M, Gonzalez FJ, Meyer UA: Oxidation of midazolam and triazolam by human liver cytochrome P450IIIA4 Mol Pharmacol 1989, 36(1):89-96.

21. Wandel C, Bocker R, Bohrer H, Browne A, Rugheimer E, Martin E: Midazolam is metabolized by at least three different cytochrome P450 enzymes. Br J Anaesth 1994, 73(5):658-661.

22. Wandel C, Witte JS, Hall JM, Stein CM, Wood AJ, Wilkinson GR: CYP3A activity in African American and European American men: population differences and functional effect of the CYP3A4*1B5'-promoter region polymorphism. Clin Pharmacol Ther 2000, 68(1):82-91.

doi:10.1186/2049-6958-7-31

Cite this article as: Tulek et al:: Flexible bronchoscopy may decrease respiratory muscle strength: premedicational midazolam in focus. Multidisciplinary Respiratory Medicine 2012 7:31. 